Erratum

\title{
Erratum to "A Case Study on Stratified Settlement and Rebound Characteristics due to Dewatering in Shanghai Subway Station"
}

\author{
Jianxiu Wang, ${ }^{1,2}$ Tianrong Huang, ${ }^{2}$ and Dongchang Sui ${ }^{2}$ \\ ${ }^{1}$ Key Laboratory of Geotechnical and Underground Engineering of Ministry of Education, Tongji University, Shanghai 200092, China \\ ${ }^{2}$ Department of Geotechnical Engineering, Tongji University, Shanghai 200092, China
}

Correspondence should be addressed to Jianxiu Wang; jianxiu_wang@yeah.net

Received 9 July 2013; Accepted 27 August 2013

Copyright (c) 2013 Jianxiu Wang et al. This is an open access article distributed under the Creative Commons Attribution License, which permits unrestricted use, distribution, and reproduction in any medium, provided the original work is properly cited.

The typo error that happened in the affiliation has been modified as shown above.

An error occurred during uploading Figure 1 and the following is the corrected figure.

A complementary expression was added to the second paragraph of Section 2.1 as follows.

According to the geotechnical investigation report, the simplified geological section of Yishan Road station was shown in Figure 2 (the sandy clay (4) was actual (4) 2 in original geological report).

An error in calculation of model scale in Section 2.4 has been modified as follows.

Confined to the size of test box, the model scale was taken as $n=100$. The model box was $700 \times 900 \times 700 \mathrm{~mm}$ in width, length, and height, respectively. To measure the stratified settlement and rebound, nine displacement meters were placed as Figure 6 indicates.

An error regarding the model scale in Section 3.2.2 has been modified as follows.

According to the test scheme, the settlement of layers number 4 and number 5 in different acceleration was drawn in Figures 12 and 13, respectively (the value of SSR has multiplied with the scale 100 to make it more clear).

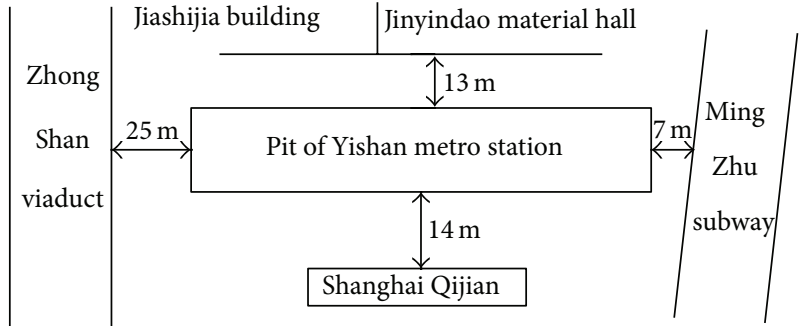

Figure 1: Layout of Yishan metro station. 

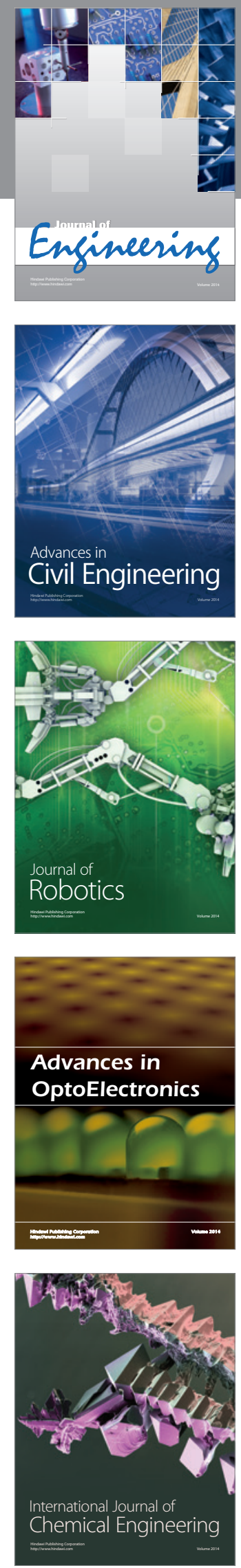

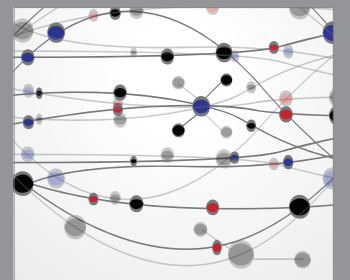

The Scientific World Journal
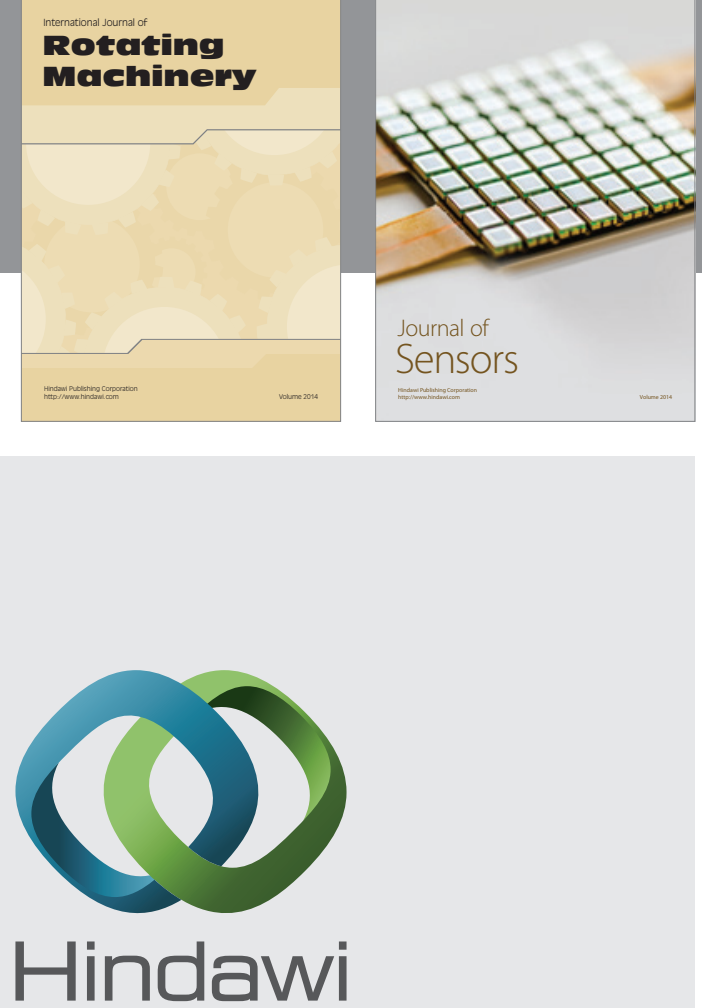

Submit your manuscripts at http://www.hindawi.com
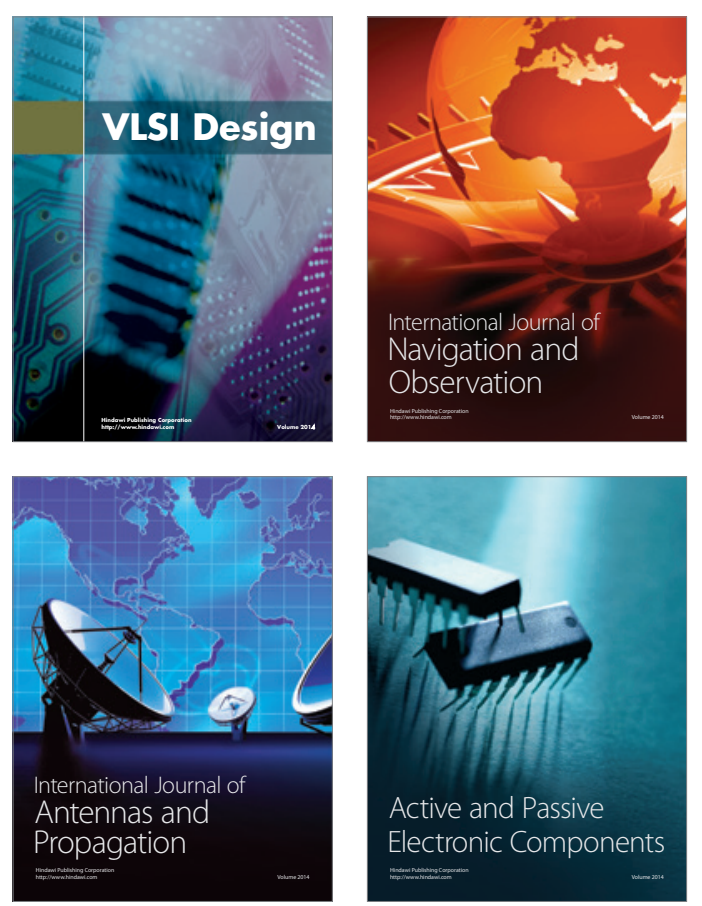
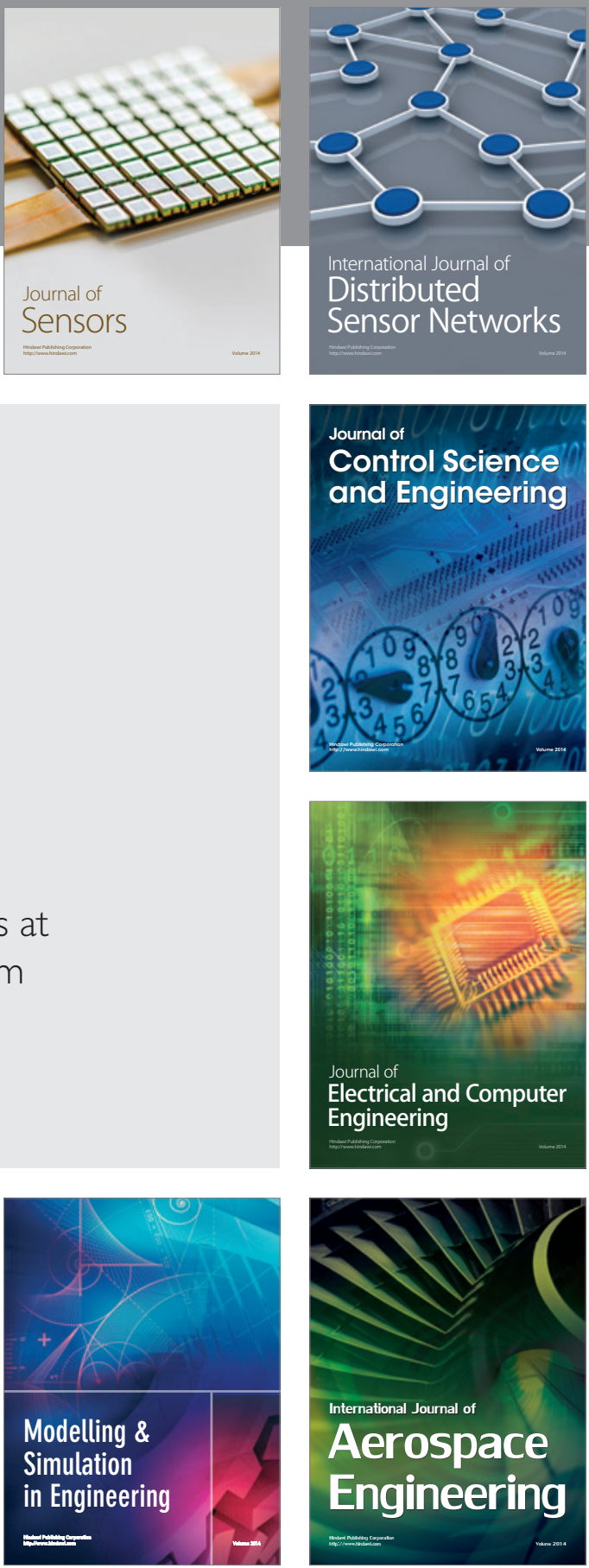

Journal of

Control Science

and Engineering
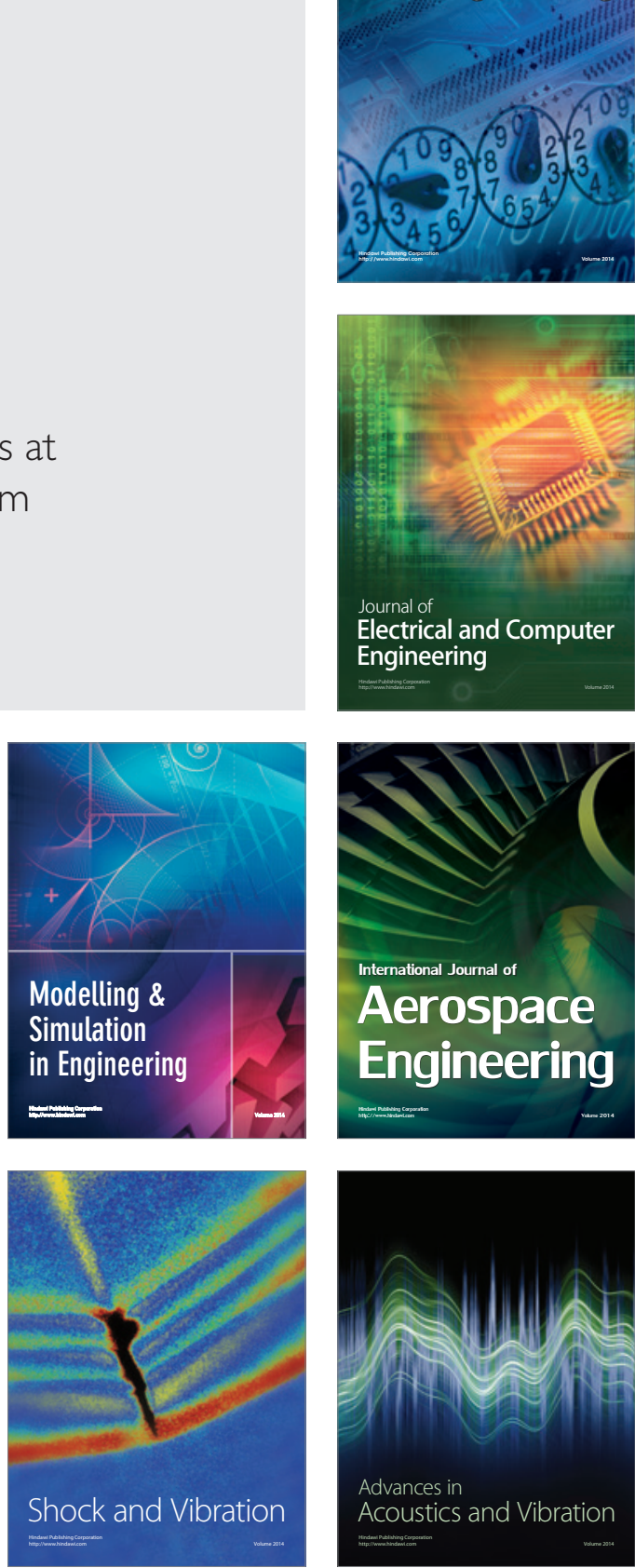